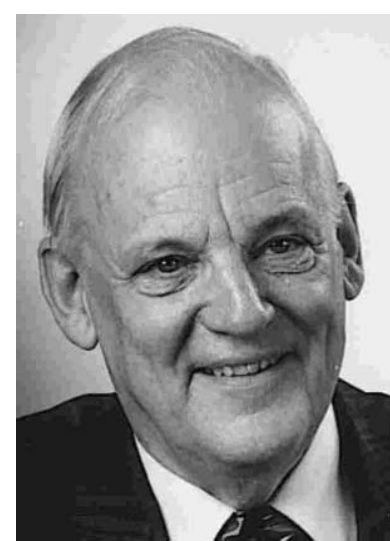

Gösta Samuelson gosta.samuelson@htu.se

\title{
Folic acid: a key vitamin in nutrition
}

$\mathrm{F}$ olic acid, present in different chemical forms of folates in the diet, is essential for cell division, biosynthesis of DNA and RNA, and growth. Furthermore, folates together with vitamin $B_{12}$, are cofactors in the metabolism of homocysteine. During the past few years the requirement of dietary folate intake has been debated. The need is particularly high before, during and after the conception to prevent neural tube defects (NTD). There are also indications of a protective effect against cardiovascular disease and associations with the development of mental degenerative disorders such as Alzheimer's disease. Knowledge of the folate content in different foods, as well as the bioavailability of folates from different foods, is essential. In this issue of the Scandinavian Journal of Nutrition, Cornelia Witthöft and co-workers describe a new method for evaluating the bioavailability of folates from foods in humans, using an ileostomy model.

\section{Methods for folate quantification in food}

Different methods for the quantification of folates in berries were evaluated in a dissertation by Lena Strålsjö (Uppsala, Sweden, 2003). Microbiological assay (MA), the method previously used most often, was compared with high-performance liquid chromatography (HPLC) and radioprotein-binding assay (RPBA). The last method was found to be the most reliable. The predominant folate form in testing of berries was $5-\mathrm{CH}_{3}-\mathrm{H}_{4}$ folate. The highest folate content was found in rose hips and strawberries. Cultivar, ripeness, year of harvest and temperature exerted an influence on the folate content. Much higher folate contents were found in berries compared with current food table data. These results are important and should be followed by a critical update of folate contents in other food groups in the Swedish Food Composition Tables.

\section{Dietary folate intake and content}

The current dietary folate intake in most Western countries is below the recommendations. The mean daily intake of folate in Sweden in men and women is only 217 and $232 \mu \mathrm{g} \mathrm{day}^{-1}$, respectively (Becker, 1999). The major contributors to folate are vegetables, fruits, berries and potatoes. In countries in which fortification is common, such as the USA and Canada, bread and cereals are also important sources. Many countries like Sweden have, however, not yet adopted that strategy.

\section{Recommendation}

Many studies published since the early 1990s have shown that it is important for women to have high folate stores from the beginning of pregnancy to avoid NTD in the baby. These studies have resulted in a recommendation to increase the folate intake to $400 \mu \mathrm{g} \mathrm{day}^{-1}$ when planning a pregnancy or early in the pregnancy, either by increasing the intake of vegetables and fruit or by taking supplements, or both.

\section{Fortification of food: why not?}

Sweden has a low incidence of NTD. This is the main reason why no decision to enrich any products has yet been taken. The situation is complicated, since too high folate concentration is reported to result in multiple births, in turn giving some more preterm births with increased a risk of perinatal and postnatal complications (Eriksson et al. Twin Res 2001; 4: 63-6, Sweden; Lumley et al. Br J Obstet Gynaecol 2001; 108: 937-42, Australia). Recently, however, a study in China (Li et al. Lancet 2003; 361: 380-4) did not confirm these results, but a low rate of dizygotic twins in China may limit the extent of these findings.

One must also remember that in the elderly fortification of flour by folic acid may mask a vitamin $B_{12}$ deficiency, leading to neurological damage. Therefore, the question of general folate fortification of flour is certainly complicated. However, whether fortification or supplementation is eventually chosen in Sweden, it is important to reach a decision on this matter. 